\title{
A Study of Five Parameter Type I Generalized Half Logistic Distribution
}

\author{
Bello Olalekan Akanji ${ }^{1}$, Sule Ibrahim ${ }^{1}$, Awodutire Phillip Oluwatobi ${ }^{2}$, Olapade Akintayo Kehinde ${ }^{1}$ \\ ${ }^{1}$ Department of Mathematics, Obafemi Awolowo University, Ile-Ife, Nigeria \\ ${ }^{2}$ Department of Statistics, Federal Polytechnic of Oil and Gas, Bonny, Nigeria
}

Email address:

olalekan4sure@gmail.com (Bello O. A.), ibrahimsule76@yahoo.com (Sule I.), phillip.awodutire@gmail.com (Awodutire P. O.), akolapad@oauife.edu.ng (Olapade A. K.)

\section{To cite this article:}

Bello Olalekan Akanji, Sule Ibrahim, Awodutire Phillip Oluwatobi, Olapade Akintayo Kehinde. A Study of Five Parameter Type I Generalized Half Logistic Distribution. Pure and Applied Mathematics Journal. Vol. 6, No. 6, 2017, pp. 177-181.

doi: 10.11648/j.pamj.20170606.14

Received: October 29, 2016; Accepted: October 23, 2017; Published: January 4, 2018

\begin{abstract}
In this paper, we obtained a generalized half logistic distribution which is called a five-parameter type I generalized half logistic distribution. The distributional properties of the model such as the cumulative distribution function (cdf), moment, skewness, kurtosis, the median and the mode of the generalized distribution were established and finally a theorem that relate the distribution to pareto distribution was stated and proved.
\end{abstract}

Keywords: Characterizations, Continuous Distribution, Exponential, Kurtosis, Skewness

\section{Introduction}

Probability distribution assigns chance of occurrence of an event to random variables. This can be discrete or continuous and the Logistic and half logistic distributions are both continuous.

The half logistic distribution which is also known as folded logistic is derived from logistic distribution by truncating the interval $(-\infty, \infty)$ at point $x=0$ of the probability density function (pdf) of logistic distribution. The pdf of half logistic distribution is given as

$$
f(x)=\frac{2 e^{x}}{\left(1+e^{x}\right)^{\wedge 2}}, \mathrm{x} \geq 0 .
$$

And its cumulative density function is given as

$$
F(x)=\frac{e^{x}-1}{1+e^{x}}, \mathrm{x} \geq 0 .
$$

One of the attractions of this distribution in the context of reliability theory is that it has a monotonically increasing hazard rate for all parameter values, a property shared by relatively few distributions which have support on the positive real half-line. In terms of tail behavior, the halflogistic distribution provides a degree of flexibility as its tail thickness lies between those of the half-normal and half-
Cauchy distributions. The half-logistic distribution has also been used successfully to model records.

Half logistic model obtained as the distribution of the absolute standard logistic variate is probability model considered by Balakrishnan [5], he established some recurrence relations for the moments and product moments of order statistics for half logistic distribution. Balakrishnan and Puthenpura obtained the best linear unbiased estimates of the location and scale parameters respectively of the half logistic distribution through linear functions of order statistics and they also tabulated the values of the variances and covariances of these estimates [6]. Balakrishnan and Wong obtained approximate maximum likelihood estimates of the location and scale parameters of the half logistic distribution with type II right censoring [7]. Balakrishnan and Hossain worked on a generalized type II version of the half logistic distribution and some properties like mean, median, mode, cumulative distribution function etc of the distribution were derived [4]. Olapade derived the pdf of type II generalized half logistic distribution with four parameters and he also obtained the cdf, the hazard function, the survival function, moment and the central tendency of the distribution [9]. Torabi and Bagheri considered an extended generalized half logistic distribution. They derived some properties of this distribution and then discussed estimation of the parameters 
using the method of moments, maximum likelihood and the new method of maximum spacing distance estimator based on complete data. They also evaluated the properties of the maximum likelihood estimators through the mean squared error (MSE), relative absolute bias and relative error and finally carried out simulation of results to show the precision of the maximum likelihood estimates for the parameters involved [12]. Olapade proved some theorems that characterized the half logistic distribution [8]. The half logistic distribution has not received much attention from researchers in terms of generalization. Olapade (2011) obtained a four parameter type I generalized half logistic distribution as

$$
f(x)=\frac{p(\lambda+1)^{\wedge} p e^{x}}{\left(\lambda+e^{x}\right)^{\wedge} p+1}, \mathrm{x} \geq 0, \mathrm{p}>0 .
$$

He also obtained the cumulative distribution function (CDF), the survival function and the hazard function, moments, the $100 \mathrm{p}$-percentage point and the mode of the distribution [10]. Olapade (2014) obtained a probability density function of the Type I generalized half logistic distribution as

$$
f(x)=\frac{{ }_{2}^{p} p e^{x}}{\left(1+e^{x}\right)^{\wedge p+1}}, \mathrm{x} \geq 0, \mathrm{p}>0 .
$$

He obtained the cumulative distribution function, moments, median, mode, $100 p$-percentage point and order statistics of the distribution and estimates the parameter of the distribution using maximum likelihood method [11].

Many authors have studied various estimators of the half logistic distribution for both censored and uncensored data. For example, see Balakrishnan and Chan [3], and Adatia [1] [2].

The rest of the paper is arranged as follows: in section 2, we presented the pdf and cdf of the five parameter type I generalized half logistic distribution. In section 3, we derived the moments, skewness and kurtosis of the distribution and presented the results in a table. In section 4 , we presented the median of the distribution. In section 5 , we presented the mode. In section 6 , we presented the $100 \mathrm{k}$-percent which can be used in Acceptance Sampling. In section 7, we stated and proved a theorem that characterizes the generalized half logistic distribution with generalized pareto distribution and finally, in section 8 , we make concluding remark.

\section{The Five Parameter Type I Generalized Half Logistic Distribution}

Theorem 1: The random variable $X=\ln \left(\frac{2 e^{x}-\lambda}{\beta}\right)$ has a type I generalized half-logistic distribution, if $\mathrm{Y}$ has an exponential distribution with parameter $p$, where $\lambda$ and $\beta>0$ are constant.

Proof: If $\mathrm{Y}$ is exponentially distributed with parameter $p$,

$$
f(y)=p e^{p y}, \mathrm{x} \geq 0, \mathrm{p}>0 .
$$

$$
X=\ln \left(\frac{2 e^{x}-\lambda}{\beta}\right)
$$

by transformation of the random variable

$$
y=\ln \left(\frac{\lambda+\beta e^{x}}{2}\right)
$$

Then,

$$
\begin{gathered}
\frac{d x}{d y}=\frac{\beta e^{x}}{\lambda+\beta e^{x}} \\
f(x ; \lambda, \beta, p)=\left|\frac{d y}{d x}\right| f(y), \\
f(x ; \lambda, \beta, p)=\frac{\beta p k e^{x}}{(\lambda+\beta)^{\wedge} p+1},
\end{gathered}
$$

The value of $\mathrm{k}$ that makes equation (8) a probability density function is

$$
k=(\lambda+\beta)^{\wedge p}
$$

Therefore

$$
f(x ; \lambda, \beta, p)=\frac{\beta p(\lambda+\beta)^{\wedge p} e^{x}}{(\lambda+\beta)^{\wedge p+1}} . x \geq 0, \lambda, \beta, p>0
$$

If we introduce the location parameter $\mu$ and the scale parameter $\sigma$ in equation (9) we have

$$
f(x ; \sigma, \mu, \lambda, \beta, p)=\frac{\beta p(\lambda+\beta)^{\wedge} e^{\frac{x-\mu}{\sigma}}}{\sigma\left(\lambda+\beta e^{\frac{x-\mu}{\sigma}}\right)^{\wedge p+1}}, \quad \mathrm{x} \geq 0, \lambda, \beta, \mathrm{p}>0
$$

The pdf in equation (10) is the five parameter type I generalized half logistic distribution.

Without loss of generality, we shall assume that $\sigma=1$ and $\mu=0$.

The cdf of the five parameter type generalized half logistic distribution is given as

$$
F(x ; \lambda, \beta, p)=1-\left(\frac{\lambda+\beta}{\lambda+\beta e^{x}}\right)^{\wedge p}, \mathrm{x} \geq 0, \lambda, \beta, \mathrm{p}>0
$$

\section{Moment of the Five Parameter Type I Generalized Half Logistic Distribution}

The moment generating function is given as

$$
E(x)=\int x f(x, \lambda, \beta, p) d x
$$

Considering equation (9),

$$
E(x)=\beta p(\lambda+\beta)^{\wedge p} \int_{0}^{\infty} \frac{e^{x}}{\left(\lambda+\beta e^{x}\right)^{\wedge p+1}}
$$

The first moment of $x$ is given as

$$
E(x)=\beta p(\lambda+\beta)^{\wedge p} \int_{0}^{\frac{1}{\lambda+\beta}} \ln \left(\left(\frac{1-t \lambda}{t \beta}\right)\right)\left(\frac{1-t \lambda}{t \beta}\right) t^{\wedge p+1} \frac{d t}{t(1-t \lambda)}
$$

Similarly, the second moment of $x$ is given as 


$$
E(x)^{2}=\beta p(\lambda+\beta)^{p} \int_{0}^{\frac{1}{\lambda+\beta}}\left(\ln \left(\frac{1-t \lambda}{t \beta}\right)\right)^{2}\left(\frac{1-t \lambda}{t \beta}\right) t^{p+1} \frac{d t}{t(1-t \lambda)}
$$

Equations (14) and (15) can be evaluated numerically for various values of $\lambda, \beta$ and $p$.

Hence the mean of the five-parameter type I generalized half-logistic distribution can be computed using equation (14) and the variance of the distribution can be evaluated for each given value of $\lambda, \beta$ and $p$.

Table 1 gives the moments $\mathrm{E}\left(\mathrm{x}^{\mathrm{n}}\right)$ of the five-parameter type I generalized half logistic distribution for $\mathrm{n}=1,2,3,4$ and the variance while table 2 gives the values of skewness and kurtosis using the following relation.

$$
\mu_{1}=v_{1}, \quad \mu_{2}=v_{2}-v^{2}{ }_{1}, \mu_{3=v_{3}-3} v_{2 v_{1}}+2 v^{3}{ }_{1}, \quad \mu_{4}=v_{4-4 v_{3}} v_{1+6 v_{2}} v^{2}{ }_{1}-3 v^{4}{ }_{1}
$$

Where $v_{l}$ is the $i^{\text {th }}$ moment, $\mu_{1}=$ the mean, $\mu_{2}=$ the variance.

$$
\begin{gathered}
\beta_{1}=\frac{\mu_{3}^{2}}{\mu_{2}^{3}} \\
\beta_{2=\frac{\mu_{4}}{\mu_{2}^{2}}}
\end{gathered}
$$

Where $\beta_{1}$ is the skewness and $\beta_{2}$ is the kurtosis.

Table 1. Moment of the five-parameter type I generalized half-logistic distribution when $\lambda=1.5, \beta=2.5$.

\begin{tabular}{llllll}
\hline $\mathbf{P}$ & $\mathbf{E}(\mathbf{x})$ & $\mathbf{E}\left(\mathbf{x}^{\mathbf{2}}\right)$ & $\mathbf{E}\left(\mathbf{x}^{\mathbf{3}}\right)$ & $\mathbf{E}\left(\mathbf{x}^{\mathbf{4}}\right)$ & Variance \\
\hline 1 & 1.2533 & 2.8166 & 8.9830 & 37.1045 & 1.2458 \\
2 & 0.6756 & 0.8264 & 1.4222 & 3.1263 & 0.3699 \\
3 & 0.4682 & 0.4021 & 0.4870 & 0.7517 & 0.1829 \\
4 & 0.3597 & 0.2398 & 0.2265 & 0.2729 & 0.1104 \\
5 & 0.2925 & 0.1599 & 0.1245 & 0.1238 & 0.0743 \\
6 & 0.2467 & 0.1145 & 0.0759 & 0.0646 & 0.0536 \\
7 & 0.2134 & 0.0862 & 0.0499 & 0.0371 & 0.0407 \\
8 & 0.1881 & 0.0673 & 0.0346 & 0.0229 & 0.0319 \\
9 & 0.1681 & 0.0539 & 0.0249 & 0.0149 & 0.0256 \\
10 & 0.1520 & 0.0443 & 0.0186 & 0.0101 & 0.0212 \\
\hline
\end{tabular}

Table 2. The skewness and kurtosis of the five-parameter type I generalized half logistic distribution when $\lambda=1.5, \beta=2.5$.

\begin{tabular}{lllll}
\hline $\mathbf{P}$ & $\boldsymbol{\mu}_{\mathbf{3}}$ & Skewness & $\boldsymbol{\mu}_{\mathbf{4}}$ & Kurtosis \\
\hline 1 & 2.3301 & 2.8080 & 11.2143 & 7.2256 \\
2 & 0.3639 & 2.6164 & 0.9211 & 6.7319 \\
3 & 0.1275 & 2.6569 & 0.2244 & 6.7080 \\
4 & 0.0608 & 2.7473 & 0.0829 & 6.8017 \\
5 & 0.0342 & 2.9578 & 0.0383 & 6.9378 \\
6 & 0.0212 & 2.9186 & 0.0204 & 7.1007 \\
7 & 0.0142 & 2.9908 & 0.0118 & 7.1235 \\
8 & 0.0099 & 3.0192 & 0.0074 & 7.2179 \\
9 & 0.0072 & 3.0899 & 0.0049 & 7.4768 \\
10 & 0.0054 & 3.0604 & 0.0033 & 7.3425 \\
\hline
\end{tabular}

\section{The Median of the Five Parameter Type I Generalized Half Logistic Distribution}

The median of the distribution is $x_{m}$ such that

$$
\int_{-\infty}^{x_{n}} f(x) d x=\frac{1}{2}
$$

$$
\begin{array}{r}
p \beta(\lambda+\beta)^{p} \int_{0}^{x_{n}} \frac{e^{x}}{\left(\lambda+\beta e^{x}\right)^{p+1}} d x=\frac{1}{2} \\
1-\frac{(\lambda+\beta)^{\mathrm{p}}}{\left(\lambda+\beta \mathrm{e}^{\mathrm{x}} \mathrm{m}\right)^{\mathrm{p}}}=\frac{1}{2}
\end{array}
$$

By solving equation 21 to get $\mathrm{x}_{\mathrm{m}}$, we have

$$
\begin{gathered}
\frac{(\lambda+\beta)^{p}}{\left(\lambda+\beta \mathrm{e}^{\mathrm{x}_{\mathrm{m}}}\right)^{\mathrm{p}}}=\frac{1}{2} \\
\frac{\lambda+\beta}{\lambda+\beta \mathrm{e}^{\mathrm{x}_{\mathrm{m}}}}=\frac{1}{\sqrt[p]{2}} \\
\sqrt[\mathrm{p}]{2}(\lambda+\beta)=\lambda+\beta \mathrm{e}^{\mathrm{x}_{\mathrm{m}}} \\
\mathrm{e}^{\mathrm{x}_{\mathrm{m}}}=\frac{\mathrm{p}_{\sqrt{2}(\lambda+\beta)-\lambda}}{\beta}
\end{gathered}
$$

Then,

$$
x_{n=} \ln \left(\frac{\sqrt[p]{2}(\lambda+\beta)-\lambda}{\beta}\right)
$$

\section{The Mode of the Five Parameter typeI Generalized Half Logistic Distribution}

The mode of a probability density function is obtained by equating the derivative of the density function to zero and solve for the variable. Therefore, for five parameter type I generalized half logistic distribution,

$$
f(x ; \lambda, \beta, p)=\frac{\beta p(\lambda+\beta)^{p} e^{x}}{\left(\lambda+\beta e^{x}\right)^{p+1}}
$$

$\mathrm{f}^{\prime}(\mathrm{x} ; \lambda, \beta, \mathrm{p})=\beta \mathrm{p}(\lambda+\beta)^{\mathrm{p}}\left[\frac{\left(\lambda+\beta \mathrm{e}^{\mathrm{x}}\right)^{\mathrm{p}+1} \mathrm{e}^{\mathrm{x}}-\mathrm{e}^{\mathrm{x}}(\mathrm{p}+1)\left(\lambda+\beta \mathrm{e}^{\mathrm{x}}\right)^{\mathrm{p}} \beta \mathrm{e}^{\mathrm{x}}}{\left(\lambda+\beta \mathrm{e}^{\mathrm{x}}\right)^{2 \mathrm{p}+2}}\right]$

By equating the derivatives to zero, we have

$$
\begin{gathered}
\left(\lambda+\beta \mathrm{e}^{\mathrm{x}}\right)-(\mathrm{p}+1) \beta \mathrm{e}^{\mathrm{x}}=0 \\
\lambda=\mathrm{p} \beta \mathrm{e}^{\mathrm{x}} \\
\mathrm{e}^{\mathrm{x}}=\frac{\lambda}{\mathrm{p} \beta} \\
\mathrm{x}=\ln \left(\frac{\lambda}{\mathrm{p} \beta}\right)
\end{gathered}
$$

\section{100k-Percentage Point of the Five Parameter type I Generalized Half Logistic Distribution}

We obtain the 100k-percentage point from

$$
F\left(x_{x}\right)=k
$$

Where $F(x)$ is the cdf of the five parameter type I generalized half logistic distribution.

This implies

$$
1-\frac{(\lambda+\beta)^{\wedge p}}{\left(\lambda+\beta e^{x_{k}}\right)^{\wedge p}}=k
$$




$$
\begin{gathered}
\frac{(\lambda+\beta)^{\mathrm{p}}}{\left(\lambda+\beta \mathrm{e}^{\mathrm{x}} \mathrm{k}\right)^{\mathrm{p}}}=1-\mathrm{k} \\
\frac{(\lambda+\beta)}{\left(\lambda+\beta \mathrm{e}^{\mathrm{x}} \mathrm{k}\right)}=\sqrt[\mathrm{p}]{(1-\mathrm{k})} \\
\lambda+\beta \mathrm{e}^{\mathrm{x}_{\mathrm{k}}}=\frac{(\lambda+\beta)}{\sqrt[\mathrm{p}]{(1-\mathrm{k})}} \\
\beta \mathrm{e}^{\mathrm{x}_{\mathrm{k}}}=\frac{(\lambda+\beta)}{\sqrt[\mathrm{p}]{(1-\mathrm{k})}-\lambda} \\
\mathrm{e}^{\mathrm{x}_{\mathrm{k}}}=\frac{1}{\beta}\left[\frac{(\lambda+\beta)}{\mathrm{p} \sqrt{(1-\mathrm{k})}}-\lambda\right] \\
\mathrm{x}_{\mathrm{k}}=\ln \left(\frac{1}{\beta}\left[\frac{(\lambda+\beta)}{\mathrm{p} \sqrt{(1-\mathrm{k})}}-\lambda\right]\right)
\end{gathered}
$$

\section{Characterization of Five Parameter typeI Generalized Half Logistic Distribution}

Theorem 2: The random variable $y=\ln \left(\frac{2 x-\lambda}{\beta}\right)$ is a generalized half logistic variable, if $x$ follows a generalized Pareto distribution with parameter $\mathrm{p}$, where $\lambda$ and $\beta$ are positive constants.

Proof: If $x$ follows a generalized Pareto distribution with parameter $p$, then

$$
f(x ; p)=\frac{p}{x^{p+1}}, \mathrm{x}>1, \mathrm{p}>0
$$

Since the random variable

$$
y=\ln \left(\frac{2 x-\lambda}{\beta}\right) \Rightarrow x=\left(\frac{\lambda+\beta e^{x}}{2}\right)
$$

Hence,

$$
f(x ; \lambda, \beta, p)=\frac{\beta p(\lambda+\beta)^{\wedge p} e^{x}}{(\lambda+\beta)^{\wedge p}}, \mathrm{x} \geq 0, \lambda, \beta, \mathrm{p}>0
$$

Where $(\lambda+\beta)^{\wedge p}=k$

Conversely, if $\mathrm{Y}$ has a five-parameter type I generalized half logistic distribution with distribution shown in equation (27), then the cdf of the random variable $x$ is

$$
\begin{array}{r}
F(x)=p r\left[\frac{\lambda+\beta e^{y}}{2} \leq x\right]=F\left[\ln \left(\frac{2 x-\lambda}{\beta}\right)\right] \\
F(x)=1-\left(\frac{\lambda+\beta}{2}\right) \wedge p_{x}-p
\end{array}
$$

And

$$
f(x)=\left(\frac{\lambda+\beta}{2}\right)^{\wedge p} \frac{p}{x^{p+1}}
$$

By omitting the constant in equation (30) the density of $x$ can be written as

$$
g(x) \propto \frac{p}{x^{p+1}}
$$

Since any density function proportional to the right hand side of equation (46) is that of a generalized Pareto random variable.

\section{Conclusion}

This paper deals with a typeI generalized half logistic distribution. In this paper, we presented a five parameter type I generalized half logistic distribution and proved that it is a pdf. We established the moments and used it to obtain the mean, variance, skewness and kurtosis by presenting the results in tables 1 and 2 . We obtained the median, mode and $100 p$-percent point which can be applied in acceptance sampling. We also stated and proved a theorem that relates this distribution to pareto distribution.

From equation (9) when $\beta=1$, it reduces to equation (3) of Olapade 2011, when $\lambda=\beta=1$, it reduces to equation (4) of Olapade 2014 and when $\lambda=\beta=\mathrm{p}=1$ it reduces to the standard logistic distribution. Further research can be to characterize the distribution with other statistical distributions and the area of applications can also be taken in to account.

\section{References}

[1] Adatia, A. (1997). Approximate BLUE's of the parameters of the half logistic distribution based on fairly large doubly censored samples. Computational Statistics and Data Analysis 24, 179-191.

[2] Adatia, A. (2000). Estimation of the parameters of the halflogistic distribution using generalized rank set sampling. Computational Statistics and Data Analysis 33, 1-13.

[3] Balakrishnan, N., Chan, P. S. (1992). Estimation for the scaled half logistic distribution under type II censoring. Computational Statistics and Data Analysis 13,123-131.

[4] Balakrishnan, N. and Hossain, A. (2007), Inference for the Type II generalized logistic distribution under progressive Type II censoring, Journal of Statistical Computation and Simulation, 77 (12), pp1013-1031.

[5] Balakrishnan, N. (1985). Order statistics from the half logistic distribution, Journal of statistical computation and simulation. vol. 20, pp.287-309.

[6] Balakrishnan, N and Puthenpura, S. (1986). Best linear unbiased estimation of location and scale parameters of the half logistic distribution, J. Statist. Comput. Simul. VOL 25. pp193-204.

[7] Balakrishnan, N. and Wong, K. H. T. (1991). Approximate MLEs for the location and scal eparameters of the half logistic distribution with Type-II right censoring, IEEE Trans. On Reliab. Vol 40. pp140-145.

[8] Olapade, A. K. (2003). On Characterizations of the Half Logistic Distribution. InterStat, February Issue, Number 2.

[9] Olapade, A. K. (2009). On a four parameter type II generalized half logistic distribution. Proc. Jangjeon Math. Soc. Korea, Volume 12 (1), pp.21-30.

[10] Olapade, A. K. (2011). On a four-parameter type I generalized half logistic distribution. Proceeding of the Jangjeon Mathematical KOREA. Vol 14. pp189-198.

[11] Olapade, A. K. (2014). The type I generalized half logistic distribution, JIRSS. Vol 13. pp 69-82. 
[12] Torabi, H. and Bagheri, F. K. (2010). Estimation of parameter for an extended generalized half-logistic distribution based on complete and censored data, JIRSS. Vol 9. pp171-195. 